\title{
超导隧道结在事件视界望远镜黑洞成像及 射电天文中的应用
}

史生才 ${ }^{1,2^{*}}$, 李婧 1,2

1. 中国科学院紫金山天文台, 南京 210034;

2. 中国科学院射电天文重点实验室, 南京 210034

*联系人, E-mail: scshi@pmo.ac.cn

\section{Superconducting tunnel junctions for black-hole imaging by Event Horizon Telescope and for radio astronomy}

\author{
Shengcai Shi ${ }^{1,2^{*}} \&$ Jing $\mathrm{Li}^{1,2}$ \\ ${ }^{1}$ Purple Mountain Observatory, Chinese Academy of Sciences, Nanjing 210034, China; \\ ${ }^{2}$ Key Lab of Radio Astronomy, Chinese Academy of Sciences, Nanjing 210034, China \\ * Corresponding author, E-mail: scshi@ pmo.ac.cn \\ doi: 10.1360/TB-2019-0073
}

2019年4月10日，事件视界望远镜(Event Horizon Telescope, EHT $)^{[1]}$ 发布了人类历史上第一张真实的黑洞照片. 此次EHT观测集合了横跨四大洲的 8 台射电天文望远镜(包 括JCMT(James Clerk Maxwell Telescope)等6台单天线望远 镜和SMA(Submillimeter Array)与ALMA(Atacama Large Millimeter/submillimeter Array)两台干涉阵), 观测波段在 $1.3 \mathrm{~mm}$ (即 $230 \mathrm{GHz}$ 频率). 基于甚长基线干涉阵(very long baseline interferometer, VLBI)技术形成了一个口径相当于 地球直径的单天线望远镜, 使其空间分辨率达到天文观测 前所未有的 20 微角秒. 需要指出的是, 具有最大口径和最 高灵敏度的ALMA干涉阵在此次射电星系M87中心黑洞观 测中发挥了决定性作用. 毫米波段VLBI技术的应用实现 了超高空间分辨率, 而决定探测灵敏度的主要因素则是上 述望远镜及阵列所配备的毫米波超导隧道结混频器 ${ }^{[2]}$. 实 际上, 正是由于超导隧道结混频器技术的诞生, 毫米波/亚 毫米波射电天文学才得以快速发展, 迄今为止地面最大望 远镜ALMA的建设才成为现实. 本文第一作者及其合作 者 ${ }^{[3]}$ 早年发展的并联双子超导隧道结技术 (parallel connected twin junctions, PCTJ)被应用于ALMA, 其研究团队 与日本国立天文台合作承担了ALMA第八和第十波段超导 隧道结混频器的研制. 此外, 还带领中国科学院紫金山天 文台和中国台湾 “中研院” 天文与天体物理研究所 (Academia Sinica Institute of Astronomy and Astrophysics, ASIAA)相关研究团队, 共同研制了ASIAA与美国哈佛-史
密松天体物理中心合作建设的SMA干涉阵的部分超导隧 道结混频器.

超导隧道结一般由两块面积在平方微米尺度的超导体 和纳米尺度厚的中间势垒层构成, 简称SIS(superconductorinsulator-superconductor) 或 STJ(superconducting tunnel junction), 结构上类似于一个电容, 但具有超低暗电流和超强 非线性电压-电流特性两大特征(图1). 混频器的功能则是 将被探测微弱信号与一个相近频率的本地高频率稳定度 参考信号差频, 产生一个中频信号, 再进行低噪声放大和 频谱处理. 得益于超导隧道结的奇异特性, 毫米波/亚毫米 波段超导隧道结混频器实现了以往半导体肖特基二极管 混频器所不可比拟的性能, 灵敏度逼近测不准原理制约的 量子极限 (即 $h v /\left(2 k_{\mathrm{B}}\right), h$ 是普朗克常数, $v$ 是探测频率, $k_{\mathrm{B}}$ 是 玻尔兹曼常数). 经过二十多年的发展, 特别是在国际大科 学装置ALMA及SMA和Herschel空间天文台等应用驱动下, 超导隧道结混频器在0.1 1 THz频段 (300 $\mu \mathrm{m} 3 \mathrm{~mm}$ 波长) 实 现了超高灵敏度(噪声温度达 $3 h v / k_{\mathrm{B}}$ ), 广泛应用于毫米波/ 亚毫米波望远镜及干涉阵, 推动了毫米波/亚毫米波天文 学的快速发展.

众所周知，超导现象发现于1911年．但直到1957年, 基于微观量子理论的BCS理论建立, 才较为完满地解释了 超导电性的物理本质. BCS理论引人了Cooper对概念, Cooper对两个电子间的相干长度大约在1 100 nm区间, 结 合能量(即能隙)在 $m e V$ 水平. 由于能隙超低, 超导体一直 


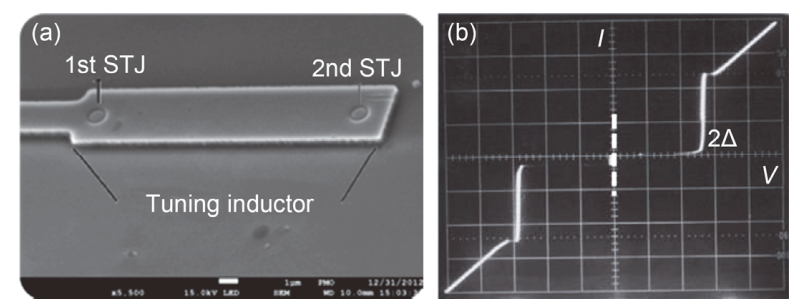

图 1 (a) 超导隧道结混频器芯片局部照片. 第 1 和第 2 个 STJ 与中 间的 Tuning inductor 构成 PCTJ 片上集成谐振腔, 两个超导隧道结的 面积约为 $1 \mu \mathrm{m}^{2}$. (b) 典型的 $\mathrm{Nb}$ 基超导隧道结在液氦温区实测直流电 压-电流 $(V-I)$ 特性. 横坐标为电压 $(1 \mathrm{mV} / \mathrm{div})$, 纵坐标为电流 $(0.1$ $\mathrm{mA} / \mathrm{div})$. 零电压处标示虚线为源于 Cooper 对隧穿效应的直流 Josephson 电流; 在超导能隙 $(2 \Delta)$ 以下, 源于热激发准粒子的暗电流 几乎为零; 在超导能隙处的电流源于准粒子隧穿效应 (因为超导能隙 处态密度接近无穷大, 所以显示突变) ; 在超导能隙以上, 显示了正 常态电流(因为态密度趋于恒定值, 所以电阻不变)

Figure 1 (a) Photo of a superconducting tunnel junction mixer chip (part), with the 1st STJ and 2nd STJ and the tuning inductor combined as an on-chip integrated resonance cavity called PCTJ. The area of the superconducting tunnel junctions is about $1 \mu \mathrm{m}^{2}$. (b) Typical $I$ - $V$ curve of a $\mathrm{Nb} / \mathrm{Al}-\mathrm{AlO}_{x} / \mathrm{Nb}$ superconducting tunnel junction measured at $4.2 \mathrm{~K}$, with horizontal axis as voltage $(1 \mathrm{mV} / \mathrm{div})$ and vertical axis as current $(0.1 \mathrm{~mA} / \mathrm{div})$. The dashed line at zero voltage represents the DC Josephson current. The current below the energy gap voltage mainly due to thermally excited particles is nearly equal to zero, while there is an onset at the energy gap voltage due to nearly infinite density of states. The current increases linearly with the voltage above the energy gap voltage because of constant density of states

被认为是理想的光子探测器. 20 世纪60年代初期, Joseph-

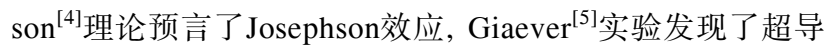
体中的准粒子隧穿效应 (二人因此与Leo Esaki共同获得 1972年诺贝尔物理学奖), 自此才真正开始了基于超导隧 穿效应的混频实验研究. 早期的混频实验研究主要聚焦于 Josephson效应, 但实验研究发现, 尽管非线性混频效应非 常显著, 混频器噪声却远超量子噪声. 主要原因是为确保 交流Josephson电流免于被结电容短路, 需要将超导隧道结 制备成点接触式(即结电容趋于零), 导致高频信号的高次 谐波混频引入显著噪声. 20 世纪 70 年代末至 80 年代初, 该 领域研究转向基于光子辅助准粒子隧穿效应 (即在一定直 流偏置电压下, 吸收 $n$ 个光子导致超导体中Cooper对被拆 散, 进而形成准粒子隧穿) 的混频器实验及理论研究. 早期 实验即显示了令人鼓舞的结果, Tucker和Feldman ${ }^{[2]}$ 则建立 了基于光子辅助准粒子隧穿效应的量子混频理论, 并预言 混频器噪声可达量子噪声、可实现变频增益, 以及具有负 阻效应等重要结果. 1983年, Bell实验室的Gurvitch等人 ${ }^{[6]}$ 发 明了基于标准光刻工艺的 $\mathrm{Nb} / \mathrm{Al}-\mathrm{AlO}_{x} / \mathrm{Nb}$ 超导隧道结制备 工艺, 使得超导隧道结器件制备可靠性及质量得到大幅提 升. 后续毫米波及亚毫米波超导隧道结混频器技术的长足 进步主要得益于两大关键技术突破: 超导隧道结片上集成 谐振和无调谐超宽带混频腔. 超导隧道结片上集成谐振技
术主要解决超导隧道结电容对高频信号的短路问题. 3种 主流的片上集成谐振技术分别为并联电感型、串联电感型 和前述 PCTJ, 其中 PCTJ兼具高输人电阻和超宽带特性. 另一方面, 早期研制的超导隧道结混频器需依赖机械调谐 装置实现望远镜观测信号与超导隧道结之间的高效耦合， 机械调谐机构设置及其在望远镜上的应用都极为繁琐. 因 此，难以应用于多频段同时观测的望远镜，更不用说多台 望远镜组成的干涉阵(如SMA和ALMA). 本文第一作者提 出了一种集成中频/直流回路的波导型超导隧道结混频腔, 通过解构及重组其不同尺度结构, 在早期有限计算能力和 软件支撑条件下完成了电磁场信号输运的高精度数值模拟 仿真, 率先实现了无调谐超宽带混频腔技术.

超导隧道结混频器在我国射电天文中的应用始于 1998 年, 即为我国青海 $13.7 \mathrm{~m}$ 毫米波望远镜研制的 $3 \mathrm{~mm}$ 波段超 导SIS接收机 ${ }^{[3]}$. 其灵敏度较之同频段半导体接收机提高 1 个量级，使得该望远镜灵敏度跻身国际前列，也使我国天 文观测从此拥有了国际先进的超导探测器. 后续还为该望 远镜研制了 $3 \mathrm{~mm}$ 波段的多谱线和多波束超导 SIS 接收机, 使 $13.7 \mathrm{~m}$ 毫米波望远镜的综合性能得到进一步提升, 目前 正在开展银道面分子谱线巡天计划“银河画卷”. 迄今为止, 国内外毫米波/亚毫米波波段射电天文望远镜仍采用经典 的 $\mathrm{Nb}$ 基超导隧道结混频器, 但其工作频率上限(约为 0.7 $\mathrm{THz}$ )受限于 $\mathrm{Nb}$ 的超导能隙. 在0.7 1.4 THz的频率区间, $\mathrm{Nb}$ 基超导隧道结尚能工作，但需结合高能隙超导或金属薄膜 构建片上集成谐振电路, 实际上频率上限只达 $1 \mathrm{THz}$. 因 此, 人们也一直在尝试研究高能隙超导隧道结及其混频器 技术. 针对高能隙超导隧道结混频器, 主要的技术挑战在 于常温条件下高质量外延超导薄膜生长、更薄势垒层的生 长, 以及更高临界电流密度下超导隧道结暗电流控制等, 利用日本国立通讯研究所的氮化铌 $(\mathrm{NbN})$ 基超导隧道结制 备工艺, 中国科学院紫金山天文台研究团队研制了 0.5 $\mathrm{THz}$ 频段 $\mathrm{NbN}$ 超导隧道结混频器, 性能达到经典 $\mathrm{Nb}$ 基超导 隧道结水平, 并实验验证了液氦以上温区 $(10 \mathrm{~K})$ 的高灵敏 度特性. 该超导隧道结混频器应用于我国的POST(Portable Submillimeter Telescope)亚毫米波望远镜, 首次实现了 $\mathrm{NbN}$ 基超导隧道结的天文观测应用 ${ }^{[7]}$, 未来将搭载我国载 人航天工程的 $2 \mathrm{~m}$ 多功能光学设施实现空间天文和大气观 测. 同时，还在发展基于 $\mathrm{Nb} / \mathrm{NbN}$ 基混合超导隧道结混频 器, 将应用于拟建的中国南极昆仑站天文台 $5 \mathrm{~m}$ 太赫兹望 远镜(DATE5)和规划中的 $60 \mathrm{~m}$ 大口径亚毫米波望远镜.

超导隧道结已经广泛应用于毫米波/亚毫米波射电天 文, 未来仍将在这一研究领域发挥重要作用. 为适应新的 科学需求, 更高能隙、更高临界电流密度超导隧道结技术, 以及超宽带、量子极限灵敏度超导隧道结混频器技术将是 未来的主要发展方向. 事件视界望远镜为进一步提升空间 分辨率，将在亚毫米波段以及空间开展黑洞观测，我国将 
建的南极DATE5望远镜、空间 $2 \mathrm{~m}$ 多功能光学设施以及未 来的大口径亚毫米波望远镜预期可以发挥重要作用. 在超 导SIS混频器技术以外, 中国科学院紫金山天文台研究团 队还发展了太赫兹超导HEB(hot-electron bolometer)混频器技 术和大规模阵列超导 TES(transition edge sensor)及MKIDs (microwave kinetic inductance detectors)探测器技术. 其中 超导SIS 和HEB混频器针对高分辨率光谱观测, 而超导TES 和MKIDs探测器针对大区域成像以及超宽带成像光谱观 测, 均为我国亚毫米波及太赫兹天文学发展以及原初引力 波探测等的关键技术.

\section{参考文献}

1 The EHT Collaboration. First M87 Event Horizon Telescope results. I. The shadow of the supermassive black hole. Astrophys J, 2019, 875: L1

2 Tucker J R, Feldman M J. Quantum detection at millimeter wavelengths. Rev Mod Phys, 1985, 57: 1055-1113

3 Shi S C, Noguchi T, Inatani J. A 100-GHz fixed-tuned waveguide SIS mixer exhibiting broad bandwidth and very low noise temperature. IEEE Trans Appl Supercond, 1997, 7: 3850

4 Joesphson B D. Possible new effects in superconductive tunneling. Phys Lett, 1962, 1: 251-253

5 Giaever I. Electron tunneling between two superconductors. Phys Rev Lett, 1960, 5: 464-466

6 Gurvitch M, Washington M A, Huggins H A. High quality refractory Josephson tunnel junctions utilizing thin aluminum layers. Appl Phys Lett, 1983, 42: 472-474

7 Li J, Takeda M, Wang Z, et al. Low-noise $0.5 \mathrm{THz}$ all-NbN superconductor-insulator-superconductor mixer for submillimeter wave astronomy. Appl Phys Lett, 2008, 92: 222504 